\title{
ARTICLE
}

\section{Estimation of Concentrations and Committed Effective Doses from Urine Samples of Nuclear Medicine Workers in Korea Due to Inhalation of ${ }^{131} \mathbf{I}$}

\author{
Gyu Hwan JEONG ${ }^{1,2, *}$, Han Ki JANG ${ }^{2}$, Joo Yeon $\mathrm{KIM}^{2}$ and Jai Ki LEE ${ }^{2}$ \\ ${ }^{1}$ Korea Institute of Nuclear Safety, 34, Gwahak-ro, Yuseong, Daejeon 305-338, Korea \\ ${ }^{2}$ Hanyang University, 17, Haengdang, Seongdong, Seoul 133-791, Korea
}

\begin{abstract}
The samples of the urine for 25 persons working in Korean nuclear medicine of the three major Korean hospitals were chosen for performing this study and ${ }^{131} \mathrm{I}$ concentrations in them were measured with high purity germanium detector. The results of the concentrations showed that the arithmetic mean of the sample was to be $66.5 \mathrm{~Bq} \cdot \mathrm{kg}^{-1}$ and the range of them to be varied from ND (non detected) to $5,045 \mathrm{~Bq} \cdot \mathrm{kg}^{-1}$. It is inferred that the worker taking the highest concentration has chronically breathed the contaminated air during the distribution job of ${ }^{131}$ I source. The committed effective doses for those were also estimated based on the measured concentrations by using IMBA software. It showed that the arithmetic mean of the dose due to inhalations of ${ }^{131} \mathrm{I}$ was to be $0.275 \mathrm{mSv}$ and the range of them to be from 0 to $4.74 \mathrm{mSv}$. The committed effective doses were to be within $2 \mathrm{mSv}$ except for the worker for distribution of ${ }^{131} \mathrm{I}$ ampule.
\end{abstract}

KEYWORDS: internal dosimetry, ${ }^{131}$ I, committed effective dose, nuclear medicine, urine sample, high purity germanium detector

\section{Introduction}

The radiation exposures in medical field are increasing as Korea goes forward to an advanced country due to the increases of the interest in the human health, the demands for the medical treatments and the application frequencies of the medical radiation. Theses increases of the demands in the medical radiation fields are resulted to increasing the occupational radiation doses for the worker members in the same fields and requiring a quantitative estimation for the internal doses of the members. But, a trial for estimating the internal doses for the members has not been presented. Though the risk due to the internal doses should be especially cared about the members in the nuclear medicines, a bioassay has not been tried out for these members in Korea According to, 2002-20, the notice of Ministry of Education, Science and Technology in Korea, which is "the regulations for measuring and deriving the internal radiation doses", a series of the process for the internal doses and the committed effective doses should be performed for the radiation workers expected to be excess of the annual dose of $2 \mathrm{mSv}^{1)}$. Especially, a periodical inspection is recommended for the workers participated in the handling task of ${ }^{131} \mathrm{I}$ in quantity by $\mathrm{ICRP}^{2}$. Based on the these reasons described in this introduction, ${ }^{131} \mathrm{I}$ was selected as the source for estimating the internal doses for the radiation walkers, because the risk due to its usage of the large quantity is expected to be very serious for the members as well as the nuclide is recognized as the very important nuclide in the field of the internal dosimetry. The urine samples of the members were employed as the examining method. ${ }^{131} \mathrm{I}$ in the urine samples was then measured by using high purity Germanium analyzer and its concentrations were also measured through

*Corresponding Author, E-mail:jkhwan@kins.re.kr

(C) Atomic Energy Society of Japan this process. Finally, estimating the committed effective doses based on the concentrations were tried out through this study.

\section{Materials and Method}

\section{Measuring the ${ }^{131} I$ content}

In this study, the ${ }^{131} \mathrm{I}$ concentrations in the urine samples of nuclear medicine workers were measured using a high purity Germanium gamma radionuclide analyzer, and the workers' doses of internal exposure to ${ }^{131} \mathrm{I}$ were evaluated with an IMBA (Integrated Modules for Bioassay Analysis) Expert Code ${ }^{6}$, which is a commercial software, a series of Windows software applications, written in Visual BASIC 6.0. The IMBA code modules implement all of the International Commission on Radiological Protection's currently recommended respiratory tract, GI-tract, biokinetic and bioassay models for the selected radionuclides, for the ICRP 68 Reference Worker.

Due to the metabolic characteristics of ${ }^{131} \mathrm{I}$, the evaluation of internal exposure based on a single sample is subject to a high level of uncertainty. The urine samples were taken five times each person at weekly intervals over a period of about one month. The urine samples were collected from 25 nuclear medicine workers based in three large hospitals (10 in hospital K, 10 in hospital A, and 5 in hospital S) with relatively higher ${ }^{131} \mathrm{I}$ use. Among the medical workers were radioactive equipment operators and nurses who had direct or indirect contact with ${ }^{131} \mathrm{I}$ through the patients who were prescribed with it. The measurements were corrected to estimate the concentration at the time of sampling, taking the time elapsed between the sampling and the analysis into consideration. On arriving at the laboratory, the samples were divided into $\mathrm{U}-8$ containers of $50 \sim 80 \mathrm{~g}$ to measure the radioactivity of ${ }^{131} \mathrm{I}$ for about 5,000 seconds using a high 
purity Ge semiconductor detector. Figure 1 below shows an example of the gamma spectrum analysis of a sample.

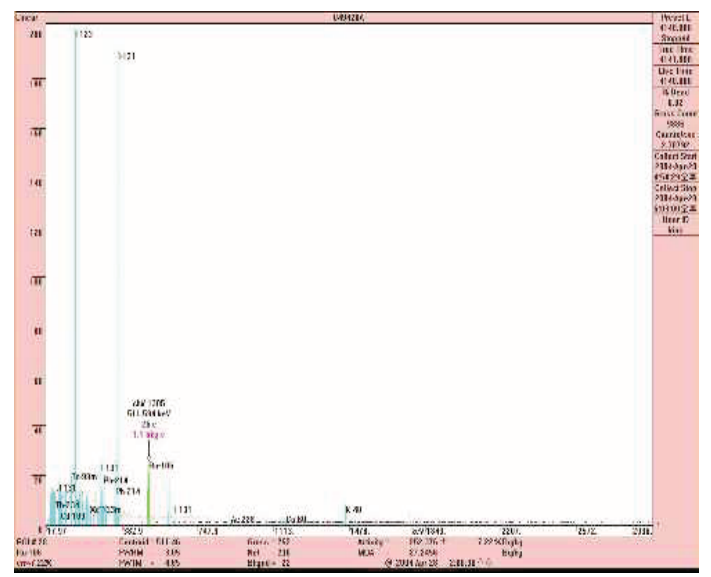

Fig. 1 An example of gamma spectrum of an urine sample

\section{Evaluation of the Committed Effective Dose}

The ${ }^{131}$ I concentration in the urine samples of the nuclear medicine workers, which were measured with a high purity Ge semiconductor detector, were converted into the concentration per daily urinary volume with which the daily intake and the committed effective dose were evaluated using the IMBA software.

For the input variables in the dose evaluation, the path and type of intake were presumed to be inhalant and chronic, respectively. The half life and the aerosol parameter of ${ }^{131} \mathrm{I}$ were set to the default values of 8.04 days and 5 Hm AMAD (Activity Median Aerodynamic Diameter) respectively. The behavior of the particles in the respiratory organs and the bio-kinetic model were applied with the default values provided in the IMBA. The dose coefficients available in the IMBA are from ICRP publications No. 68 and No. 72. In this case, where occupational exposure was evaluated, $1.1 \times$ $10^{-8}\left[\mathrm{~Sv} \cdot \mathrm{Bq}^{-1}\right]$, which corresponds to the 'Inhalation Mode" of type $F$ and $f_{1}=1$ provided in ICRP publication No. 68 , was applied as the dose coefficient ${ }^{3)}$. The urine sampling interval (days), which is an input variable for the calculation of the intakes, was set to 0.11 day considering the average frequency of urination. The average urination frequencies of people during the daytime ( 16 hours) and nighttime ( 8 hours) are 6 and 0.5 , respectively. The sampling period of 0.11 days was calculated by dividing the daytime hours with the average frequency of 6 . For the scattering factor used in the IMBA for error estimation, 1.3, which is the default value in the IDEAS Guidelines, an international standard, was applied, and the log-normal distribution which is used in the error distribution of the biological analysis was applied ${ }^{4)}$.

\section{Results and Discussion}

\section{1. ${ }^{131}$ I concentrations in Urine Samples}

A total of 121 urine samples were taken from the 25 workers on five occasions. Four samples were not available because certain workers were absent or on leave during the test period. The samples showed an average ${ }^{131} \mathrm{I}$ concentration of $66.5 \mathrm{~Bq} \cdot \mathrm{kg}^{-1}$ with a standard deviation of
$262 \mathrm{~Bq} \cdot \mathrm{kg}^{-1}$ at the time of sampling, having been corrected for the time between the sampling and the analysis, ranging from no traces at all to a maximum of $5,045 \mathrm{~Bq} \cdot \mathrm{kg}^{-1}$. The larger deviation implies that the level of internal exposure can vary depending on the characteristics of a particular job. This suggests that the level of internal exposure should be evaluated on the basis of the work itself rather than on job position. The urine sample showing the highest concentration of ${ }^{131} \mathrm{I}$ was that of the worker who actually distributed the ${ }^{131} \mathrm{I}$ and whose major pathway of exposure was via inhalation of the ${ }^{131} \mathrm{I}$ diffused into the air during distributing, despite the fact that the work was carried out under an exhaust hood. Table 1 below shows the measurements of the ${ }^{131}$ I concentration of the 25 workers.

Table $1{ }^{131}$ I concentration in urine of workers of nuclear medicine in Korea $\left(\mathrm{Bq} \cdot \mathrm{kg}^{-1}\right)$

\begin{tabular}{|c|c|c|c|c|c|c|}
\hline No. & ID & Sample 1 & Sample 2 & Sample 3 & Sample 4 & Sample 5 \\
\hline 1 & $\mathrm{O}$ & - & $10.3 \pm 1.5$ & $3.2 \pm 0.5$ & $5.8 \pm 1.1$ & $2.9 \pm 1.0$ \\
\hline 2 & A & $142 \pm 6$ & $41.9 \pm 3.1$ & $20.3 \pm 2.1$ & $123 \pm 4$ & $251 \pm 6$ \\
\hline 3 & B & $5.3 \pm 1.4$ & $3.4 \pm 1.1$ & $1.6 \pm 0.9$ & $2.7 \pm 0.5$ & $3.6 \pm 1.1$ \\
\hline 4 & $\mathrm{C}$ & $<4.8^{*}$ & $<3.3$ & $3.0 \pm 1.0$ & - & N.D. \\
\hline 5 & D & $<2.7$ & $2.8 \pm 0.6$ & $<1.8$ & N.D. & $8.4 \pm 1.5$ \\
\hline 6 & E & $9.8 \pm 1.8$ & $43.9 \pm 3.0$ & $24.8 \pm 2.9$ & $125 \pm 5$ & $26.1 \pm 1.0$ \\
\hline 7 & $\mathrm{~F}$ & $17.4 \pm 3.1$ & $14.8 \pm 0.9$ & $4.3 \pm 1.3$ & $24.6 \pm 2.4$ & $33.7 \pm 2.6$ \\
\hline 8 & G & $12.7 \pm 2.2$ & $7.4 \pm 1.2$ & $4.6 \pm 0.6$ & $5.0 \pm 1.6$ & - \\
\hline 9 & $\mathrm{H}$ & $1.9 \pm 1.2$ & $4.9 \pm 0.5$ & N.D. & $8.5 \pm 1.8$ & $22.7 \pm 2.4$ \\
\hline 10 & I & $1.3 \pm 1.5$ & $8.2 \pm 0.5$ & - & $37.3 \pm 3.0$ & $62.1 \pm 2.2$ \\
\hline 11 & N1 & N.D. & N.D. & N.D. & N.D. & N.D. \\
\hline 12 & N2 & $<4.7$ & $<2.6$ & $5.2 \pm 3.3$ & N.D. & $7.0 \pm 3.1$ \\
\hline 13 & N3 & N.D. & N.D. & N.D. & N.D. & N.D. \\
\hline 14 & N4 & N.D. & N.D. & N.D. & N.D. & N.D. \\
\hline 15 & N5 & N.D. & N.D. & N.D. & N.D. & N.D. \\
\hline 16 & N6 & $25.3 \pm 5.1$ & $13.0 \pm 5.8$ & $16.9 \pm 3.8$ & $6.1 \pm 1.2$ & $<4.6$ \\
\hline 17 & N7 & $5,045 \pm 45$ & $408 \pm 13$ & $317 \pm 8$ & $628 \pm 23$ & $191 \pm 14$ \\
\hline 18 & N8 & N.D. & N.D. & N.D. & N.D. & N.D. \\
\hline 19 & N9 & $30.1 \pm 5.9$ & $7.0 \pm 4.0$ & $3.0 \pm 2.0$ & $3.5 \pm 2.1$ & N.D. \\
\hline 20 & N10 & N.D. & N.D. & N.D. & N.D. & N.D. \\
\hline 21 & $\mathrm{~S} 1$ & $7.0 \pm 2.4$ & $57.3 \pm 5.7$ & $0.1 \pm 0.1$ & $15.6 \pm 4.2$ & $24.4 \pm 4.4$ \\
\hline 22 & $\mathrm{~S} 2$ & N.D. & N.D. & N.D. & N.D. & N.D. \\
\hline 23 & $\mathrm{~S} 3$ & N.D. & $<3.2$ & N.D. & N.D. & N.D. \\
\hline 24 & S4 & N.D. & $<3.4$ & N.D. & $<1.6$ & N.D. \\
\hline 25 & S5 & $27.1 \pm 1.3$ & $14.0 \pm 3.2$ & $10.3 \pm 2.0$ & $12.8 \pm 2.5$ & $10.0 \pm 1.9$ \\
\hline
\end{tabular}

- : No sample

* : below MDA

N.D. : Not detected

\section{Result of the Internal Dose}

The data on the ${ }^{131}$ I concentration in the urine samples of the nuclear medicine workers measured with the high purity Ge semiconductor detector were converted into the concentration per daily urinary volume, with which the daily intake and the committed effective dose were evaluated 
using the IMBA, the results of which are presented in Table 2.

Table $2{ }^{131}$ I concentration in urine of workers of nuclear medicine in Korea $\left(\mathrm{Bq} \cdot \mathrm{kg}^{-1}\right)$

\begin{tabular}{|c|c|c|c|c|c|}
\hline Hospital & No. & ID & $\begin{array}{l}\text { Intake rate } \\
\left(\mathrm{Bq} \cdot \mathrm{d}^{-1}\right)\end{array}$ & $\begin{array}{l}\text { Committed } \\
\text { effective dose } \\
(\mathrm{mSv})\end{array}$ & Duties \\
\hline \multirow{10}{*}{ A } & 1 & $\mathrm{O}$ & $1.243 \times 10^{1}$ & $4.77 \times 10^{-2}$ & IV, PET scan \\
\hline & 2 & A & $1.356 \times 10^{2}$ & $5.20 \times 10^{-1}$ & Synthesis medicines \\
\hline & 3 & B & $1.017 \times 10^{1}$ & $3.90 \times 10^{-2}$ & PET scan \\
\hline & 4 & $\mathrm{C}$ & $1.076 \times 10^{1}$ & $4.13 \times 10^{-2}$ & PET scan \\
\hline & 5 & $\mathrm{D}$ & $2.135 \times 10^{1}$ & $8.19 \times 10^{-2}$ & ${ }^{201} \mathrm{Tl}$ handling \\
\hline & 6 & E & $3.891 \times 10^{1}$ & $1.49 \times 10^{-1}$ & ${ }^{131}$ I scan \\
\hline & 7 & $\mathrm{~F}$ & $5.566 \times 10^{1}$ & $2.13 \times 10^{-1}$ & Assistant \\
\hline & 8 & G & $1.744 \times 10^{1}$ & $6.69 \times 10^{-2}$ & $\begin{array}{l}{ }^{131} \text { I uptake, } \\
\text { Heapatobiliary scan }\end{array}$ \\
\hline & 9 & $\mathrm{H}$ & $2.013 \times 10^{1}$ & $7.72 \times 10^{-2}$ & Assistant \\
\hline & 10 & I & $3.131 \times 10^{1}$ & $1.20 \times 10^{-1}$ & Tl-201 handling \\
\hline \multirow{10}{*}{$\mathrm{K}$} & 11 & N1 & 0 & 0 & Radiographer \\
\hline & 12 & $\mathrm{~N} 2$ & $1.550 \times 10^{1}$ & $5.94 \times 10^{-2}$ & Nurse \\
\hline & 13 & N3 & $1.491 \times 10^{1}$ & $5.72 \times 10^{-2}$ & Radiographer \\
\hline & 14 & $\mathrm{~N} 4$ & 0 & 0 & Radiographer \\
\hline & 15 & N5 & 0 & 0 & Radiographer \\
\hline & 16 & N6 & $2.912 \times 10^{1}$ & $1.12 \times 10^{-1}$ & Nurse \\
\hline & 17 & N7 & $1.235 \times 10^{3}$ & $4.74 \times 10^{0}$ & ${ }^{131}$ I sampling \\
\hline & 18 & N8 & 0 & 0 & Radiographer \\
\hline & 19 & N9 & $7.766 \mathrm{E}+00$ & $2.98 \times 10^{-2}$ & Nurse \\
\hline & 20 & N10 & $1.834 \times 10^{1}$ & $7.03 \times 10^{-2}$ & ${ }^{123}$ I production \\
\hline \multirow{5}{*}{$\mathrm{S}$} & 21 & S1 & $4.041 \times 10^{1}$ & $1.55 \times 10^{-1}$ & IV \\
\hline & 22 & S2 & 0 & 0 & IV \\
\hline & 23 & S3 & $1.686 \times 10^{1}$ & $6.47 \times 10^{-2}$ & Milking, IV \\
\hline & 24 & S4 & $6.576 \times 10^{0}$ & $2.52 \times 10^{-2}$ & IV \\
\hline & 25 & S5 & $5.410 \times 10^{1}$ & $2.07 \times 10^{-1}$ & $\begin{array}{c}\text { IV, oral } \\
\text { administration }\end{array}$ \\
\hline
\end{tabular}

The average and standard deviation of the committed effective dose were $0.275 \mathrm{mSv}$ and $0.937 \mathrm{mSv}$, respectively. The highest exposure dose, $4.74 \mathrm{mSv}$, was that of worker N7 from hospital $\mathrm{K}$. The hospitals in this study operate sick wards where $5.55 \times 10^{9} \sim 7.4 \times 10^{9} \mathrm{~Bq}(150 \sim 200 \mathrm{mCi})$ of ${ }^{131} \mathrm{I}$ per dosage was prescribed to each patient for oral administration. The ${ }^{131} \mathrm{I}$ had been distributed on a certain day of the week for about two hours.

In hospital $\mathrm{A}$, the highest exposure dose, $0.52 \mathrm{mSv}$, was that of the worker whose major duty was the synthesis of radioactive medicines, which was performed on an almost daily basis. No worker in hospital A showed an exposure dose exceeding $2 \mathrm{mSv}$ because the daily distributing works were shared by the workers and some of the dosages were capsulated. In hospital $\mathrm{K}$, the highest exposure dose, 4.74 $\mathrm{mSv}$, was that of the worker in charge of ${ }^{131} \mathrm{I}$ distribution. The cause of such a high concentration was attributed to the fact that the worker had carried out the distribution and repair work of the hot cell where the ${ }^{131} \mathrm{I}$ was distributed. This shows that the assignment of ${ }^{131} \mathrm{I}$ distribution work to a person leads to an increase in his or her exposure dose. In hospital S, most of the ${ }^{131} \mathrm{I}$ was delivered and used in capsules, although a certain portion was used in its liquid state. In this hospital, where ${ }^{131} \mathrm{I}$ distribution was conducted in turn by the workers, no worker showed a particularly high exposure dose. Figure 2 below is a graphic presentation of the number of workers by exposure dose.

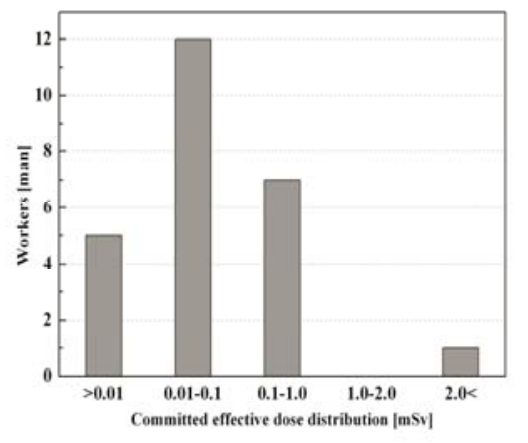

Fig. 2 Internal Dose Distribution of Nuclear Medicine Workers

\section{Determining the Optimal Number of Samples}

In the development of a policy, the major factor to consider is the distribution of the exposure dose of the population supported by the representativeness of the samples. To determine the optimal number of samples which can statistically represent the population, the following statistical assumption and formula can be used. In particular, on the assumption that the population is distributed normally, the optimal number $\mathrm{n}$ of the samples can be calculated with the equation below using the standard deviation ${ }^{5}$.

$$
\mathrm{n}>\left(\mathrm{z}_{\mathrm{a} / 2} \cdot \mathrm{s} / \mathrm{d}\right)_{2}
$$

$$
\begin{aligned}
& \text { where, } \mathrm{z}_{\mathrm{a} / 2}: 1.96 \text { for Positive False error } 5 \% \\
& \mathrm{~s}: \text { standard error } \\
& \mathrm{d}: \text { error limit }
\end{aligned}
$$

Since the criterion for personal exposure dose monitoring is $2 \mathrm{mSv}$, the accuracy of the dose should be $0.1 \mathrm{mSv}$ or a significant number to the first decimal place. In this study, the error limit was set to $0.1 \mathrm{mSv}$. The minimum number of the samples which can represent the population was calculated by applying the standard deviation of the samples as the standard error and substituting with the error limit 0.1 $\mathrm{mSv}$ into the equation. The minimum number of required samples was calculated to be 337.3 by applying $0.937 \mathrm{mSv}$ in the equation, which was the standard deviation calculated from the sample analysis as the standard error. This means 
that at least 338 samples are required to obtain statistically significant results which could represent the internal exposure dose of the worker handling ${ }^{131} \mathrm{I}$.

Although urine samples collected over 24 hours are required for a more precise evaluation, spot samples were collected for the purposes of this study. Therefore, further studies on the appropriateness of the spot samples and of their representativeness for 24 hours should be conducted by evaluating their correlation with the 24-hour samples and the degree of error. It would also be necessary to compare the results obtained from indirect methods with those from direct methods using a whole body counter or a thyroid monitor. In addition, the correlation between the atmospheric concentration and the evaluated exposure dose of ${ }^{131} \mathrm{I}$ by comparing the data would be necessary. Furthermore, analyses of urine samples collected over one full year would be required to evaluate the exposure dose, taking the air change rate of the room and seasonal variation into consideration.

\section{Conclusion}

Quantitatively evaluating the internal doses expected under the tasks related to the handling of ${ }^{131} \mathrm{I}$ by the radiation workers in the nuclear medicine of Korea is positively necessary for ensuring the health of these worker members. Therefore, this study aims at suggesting the processes for estimating the inhaled concentrations of ${ }^{131} \mathrm{I}$ from the urine samples obtained from these worker members and the committed effective doses resulted from the concentrations, and for inferring the optimized sample number. The concentrations of ${ }^{131} \mathrm{I}$ using $\mathrm{HP}(\mathrm{Ge})$ detector were varied from non-detected to $5,045 \mathrm{~Bq} \cdot \mathrm{kg}^{-1}$, and their geometric mean and standard deviation to be 66.5 and $262 \mathrm{~Bq} \cdot \mathrm{kg}^{-1}$, respectively. From these concentration results, the mean committed effective dose was to be $0.275 \mathrm{mSv}$, but the worker corresponding to the urine sample identified as the maximum concentration was turned out to directly participate in the distribution task of the radionuclide. And, his committed effective dose was to be $4.74 \mathrm{mSv}$. These dose results show that most of the radiation workers employed in the nuclear medicine are exposed as within 2
$\mathrm{mSv}$, which is recommended as the limit of the fourth article of notice 2008-51 of MEST in Korea. Since the dose for the worker participated in the task of direct distribution of the nuclide is expected to be higher than this limit, the periodically inspection and the obligation for observing the procedures for radiation safety management are required for ensuring his health.

Finally, statistically inferring the optimized sample number was tried out for ensuring the representative for the result of the doses by ${ }^{131} \mathrm{I}$ inhalation and the least 338 number was predicted to be required for the reliable result by applying the standard deviation of $0.937 \mathrm{mSv}$ and the error limit of $0.1 \mathrm{mSv}$ of the doses.

The procedure for the occupational internal dosimetry under the chronic intake of the nuclides has been established by using the urine samples obtained from the radiation workers in the nuclear medicine of Korea and $\mathrm{HP}(\mathrm{Ge})$ detector through this study. The results of concentrations, doses and optimized samples can contribute to improving the regulations and deciding the radiological policies for the internal dosimetry of the radiation workers handling the radioisotopes in the medical fields. Finally, this study can be expanded to a basic one for guaranteeing the representative or the reliability for the results of internal doses for these workers handling ${ }^{131} \mathrm{I}$.

\section{References}

1) Notice of Ministry of Education, Science and Technology No. 2008-51 (2008) "The regulations for measuring and deriving the internal radiation doses"

2) ICRP Publication 78, Individual Monitoring for Internal

Exposure of Workers: Replacement of ICRP Publication 54, Annals of the ICRP Vol. 27 No. 3-4 4-5(1998)

3) ICRP Publication 68, Dose Coefficients for Intakes of

Radionuclides by Workers" - Replacement of ICRP Publication 61, Annals of the ICRP Vol. 24 No. 4 45-46(1995)

4) www.ideas-workshop.de, The IDEAS General Guidelines for the Estimation of Committed dose from Incorporation monitoring data, IAEA (2005)

5) KIM Woo Chul, "Statistics" $4^{\text {th }}$ edition, Young Ji press, 211-215(2001).

6) www.imbaexpert.com. 